\title{
Prevalence, knowledge, attitudes and factors associated with exclusive breastfeeding among mothers in Dhaka, Bangladesh: A cross-sectional study
}

\author{
Mehedi Hasan ${ }^{1}$, Md. Nazmul Hassan ${ }^{1}$, Md. Shafiqul I. Khan², Md. Abu Tareq ${ }^{2}$, Mst. Shakila Afroj ${ }^{3}$
}

\author{
AFFILIATION \\ 1 Department of Environmental Sanitation, Patuakhali Science and \\ Technology University, Patuakhali, Bangladesh \\ 2 Department of Food Microbiology, Patuakhali Science and Technology \\ University, Patuakhali, Bangladesh \\ 3 Department of Public Health Nutrition, Primeasia University, Dhaka, \\ Bangladesh
}

CORRESPONDENCE TO

Mehedi Hasan. Department of Environmental Sanitation, Patuakhali

Popul. Med. 2021;3(September):23
Science and Technology University, Patuakhali, 8602, Bangladesh. E-mail: mehedi.nfs.pstu@gmail.com ORCID ID: https://orcid.org/0000-00024974-4057

KEYWORDS

exclusive breastfeeding, knowledge, attitude, factors, Bangladesh

Received: 11 April 2021, Revised: 10 July 2021, Accepted: 10 July 2021

https://doi.org/10.18332/popmed/140132

\begin{abstract}
INTRODUCTION Exclusive breastfeeding is balanced nutrition for growth and development of the infant, prevents stunting as well as protects from infectious and chronic diseases and has also potential to reduce infant mortality. Thus, the study aimed to assess maternal knowledge, attitudes and other factors associated with exclusive breastfeeding practices.

METHODS A cross-sectional study was conducted among 385 mothers of children aged $\leq 12$ months in Mother and Child Hospital in Dhaka city, Bangladesh, from June to December 2019 using a structured questionnaire. Data were collected by face-to-face interview from hospital's outdoors, following simple random sampling process from hospital register list. Chi-squared test and multiple logistic regression models were used to explore the association.

RESULTS The prevalence of exclusive breastfeeding was $63.4 \%$ for mothers with children aged $\leq 12$ months. Good knowledge $(\mathrm{p}<0.001)$ and positive attitude $(\mathrm{p}<0.01)$ on breastfeeding
\end{abstract}

were significantly associated with exclusive breastfeeding practices. Housewife (AOR=5.84; 95\% CI: 2.42-14.13), literate (AOR=7.16; 95\% CI: $1.3-39.36)$, family monthly income $\geq 15000$ BDT (AOR=5.41; 95\% CI: 1.78-16.47), normal delivery $(\mathrm{AOR}=13.58$; 95\% CI: 5.29-35.48) and hospital delivery (AOR=3.38; 95\% CI: 1.24-9.23) mothers were more likely to follow exclusive breastfeeding practices compared to their counterparts. Moreover, joint family $(\mathrm{AOR}=0.27$; 95\% CI: 0.12-0.62) and divorced (AOR=0.12; 95\% CI: 0.03-0.58,) mothers had less likelihood of exclusive breastfeeding practices than their counterparts.

CONCLUSIONS Good knowledge, positive attitude and some sociodemographic factors were significant with exclusive breastfeeding which are needed to improve for promoting exclusive breastfeeding practices and reducing infant mortality and morbidity.

\section{INTRODUCTION}

Exclusive breastfeeding (EBF) is considered an effective way to provide balanced nutrition, better growth, development of the infant, prevent stunting as well as protect from infectious and chronic diseases ${ }^{1}$. Simultaneously, it decreases infant morbidity and mortality due to common illnesses ${ }^{2}$ such as diarrhea or pneumonia etc. The WHO (World Health Organization) and UNICEF (United Nations Children's Emergency Fund) recommended exclusive breastfeeding for the first six months of age and giving no other food or drink to the infant, not even a single drop of water in this period ${ }^{3}$.
Globally, the exclusive breastfeeding rate is only $41 \%$ of infants under six months of age $\mathrm{e}^{4}$. The collective target for the global rate in 2030 is $70 \%$ for exclusive breastfeeding 5 . Exclusive breastfeeding rate is comparatively high in Eastern and Southern Africa (55\%), South Asia (54\%) and lower in the Middle East (30\%) and North Africa (29\%) ${ }^{6}$. Moreover, the prevalence of exclusive breastfeeding among South Asian countries was: $54.9 \%$ in India, $1.4 \%$ in China, $65.2 \%$ in Nepal, $37.7 \%$ in Pakistan, and $82.3 \%$ in Sri Lanka (WHO 2018). In Bangladesh, the prevalence of exclusive breastfeeding was about 55\% in children under six months of age ${ }^{7}$. This lower 
exclusive breastfeeding rate needs proper intervention to promote exclusive breastfeeding to achieve the targets of SDG-3 (Sustainable Development Goals) by reducing infant mortality and morbidity (minimize the neonatal mortality $<12$ and under- 5 mortality to $<25$ ) by $2030^{8}$. Worldwide, exclusive breastfeeding is considered an effective intervention for preventing infant morbidity and mortality and can prevent 1.4 million deaths among children every year ${ }^{2}$. So, neonatal and infant mortality can be reduced by increasing exclusive breastfeeding practices ${ }^{9}$.

Several factors are impeding the continuation of exclusive breastfeeding practice among lactating mothers in the world, including: 1) Geographical factors such as living area, place of delivery ${ }^{10}$; 2) Socioeconomic factors such as the education of mother ${ }^{11}$, occupation of the mother ${ }^{12}$; and 3 ) Health-related and individual factors such as mother's age, mode of delivery, the gender of the child, cultural practices, support and guidance provided by health staff or doctor, etc. ${ }^{8}$ In addition, Bangladeshi mothers face several factors that hamper them from continuing exclusive breastfeeding practices resulting in increased infant and maternal morbidity and mortality rates. Previous studies report that health facilities and delivery (hospital vs home, normal vs caesarian delivery, etc.) are associated ${ }^{3}$ with the prevalence of exclusive breastfeeding practices ${ }^{8}$. However, few studies have been conducted on risk factors associated ${ }^{12}$ with the prevalence of exclusive breastfeeding practices as well as household, environmental, and cultural factors. No studies have been conducted in Bangladesh on associations among knowledge, attitudes, and some sociodemographic factors with exclusive breastfeeding practices. As a result, there is an urgent need to evaluate knowledge, attitude, and associated sociodemographic factors with exclusive breastfeeding practice. The findings of this study will be useful to governments/NGOs (Non-Governmental Organizations) to improve policy that focuses on resources and interventions to promote exclusive breastfeeding practices that help to achieve SDG-3 (Sustainable Development Goals) and reduce infant morbidity and mortality rates. Therefore, this study has been designed to evaluate maternal knowledge, attitude and factors associated with exclusive breastfeeding among mothers who had a child aged $<12$ months.

\section{METHODS}

\section{Description of the study area}

This study was conducted in the district of Dhaka at the Mother and Child Hospital. Dhaka has a population of about 12 million. Mothers from all socioeconomic status attend this hospital due to the availability of maternal and child healthcare services. Therefore, this hospital was considered as being representative of the study population.

\section{Study design and study population}

This cross-sectional study was conducted among mothers who came to the hospital from July to December 2019. A total of 385 mothers were interviewed for the study who came to the hospital's outpatient department to get treatment related to mother and child. The inclusion criteria were women who had children aged $\leq 12$ months. Exclusion criteria were: the medical condition of the mother; medications which interrupted breastfeeding; and mothers who were diagnosed with severe congenital malformations; and those who had $>12$ months old children. These conditions were verified by using a health card which was monitored by the duty doctor at the hospital.

\section{Sample size estimation and procedure}

The minimum required sample size was calculated by using a single population proportion formula $\mathrm{n}_{\mathrm{o}}=\mathrm{Z}^{2} \mathrm{p} \mathrm{q} / \mathrm{d}^{2} ; 5 \%$ margin of error, $95 \%$ confidence intervals, and estimated to practice EBF $65 \%$ of the national rate ${ }^{13}$. This yielded a sample size of 385 with $10 \%$ non-response rate. The mothers were randomly selected from the healthcare register of the hospital and interviewed when they waited for healthcare services. If the selected mother was not interested in the study she was excluded.

\section{Data collection}

Research assistants were recruited and trained for the fieldwork of the study and collection of data by reviewing the questionnaire through teamwork with a researcher. Structured questionnaires were used to collect data. A random sampling approach was adopted to collect information like knowledge, attitude, and some sociodemographic factors through face-to-face interviews.

\section{Questionnaire}

The questionnaire involved some general information questions like sociodemographic information including name, age, education level, occupation, living area, economic status, religion, place, and types delivery, etc.

To ascertain maternal knowledge on exclusive breastfeeding, a set of questions was developed. Knowledge on exclusive breastfeeding was measured by asking the mother 10 questions such as benefits of exclusive breastfeeding and colostrum, approach of handling breastfeeding during pregnant condition of mother and infant with diarrhea disease, and other questions about advantages of breastfeeding both for infant and mother etc. Each correct answer was given a score of 1 , while a wrong answer was given a score of 0 . The total knowledge score was then calculated and graded on a scale based on standards carried out in previous research, as follows: Good ( $\geq 7$ ), medium (4-6) and poor $(\leq 3)^{14}$.

In the same way, attitude toward breastfeeding was assessed using a series of questions such as exclusive breastfeeding is beneficial for infant and mother, baby can survive without water up to 6 months of age, formula milk is unsuitable for baby and not a better choice for working mothers, and breast milk is more easily digested than formula milk. If the mother agreed with this opinion she was 
given a score of one and 0 if she disagreed or was neutral with the opinion. The total score was then calculated and the attitude was graded as: positive ( $\geq 7$ ), moderate (4-6) or poor $(\leq 3)^{14}$.

\section{Statistical analysis}

Statistical analyses were performed using IBM SPSS (version 23). Means and standard deviation (SD) were used for numerical data, whereas percentages were used for categorical data. Chi-squared test was used to assess the association of knowledge and attitude on breastfeeding with exclusive breastfeeding practices. Multiple logistic regression analyses were done to assess sociodemographic factors on exclusive breastfeeding practices. The model fitness was tested using Hosmer and Lemeshow test. The adjusted odds ratio (AOR) was calculated to evaluate the strength of the association of sociodemographic factors with exclusive breastfeeding practices at $95 \% \mathrm{CI}$ for the significance test. A value of $\mathrm{p}<0.05$ was considered statistically significant.

\section{RESULTS}

Table 1 shows the sociodemographic and other characteristics of respondents. In this study population, more than half $(54.5 \%)$ of the mothers were aged $\geq 24$ years, with a mean $( \pm S D)$ age of $26.1( \pm 5.3)$ years. In regard to education level, $17.2 \%$ respondents were illiterate, $18.7 \%$ were primary

Table 1. Sociodemographic and other characteristics of mothers in the study $(\mathrm{N}=385)$

\begin{tabular}{|c|c|c|c|c|}
\hline \multirow[t]{2}{*}{ Characteristics } & \multirow{2}{*}{$\begin{array}{l}\text { Total } \\
\text { n (\%) }\end{array}$} & \multicolumn{2}{|c|}{ Exclusive breastfeeding } & \multirow[t]{2}{*}{$\mathbf{p}^{*}$} \\
\hline & & $\begin{array}{c}\text { Yes } \\
\text { n (\%) }\end{array}$ & $\begin{array}{c}\text { No } \\
\text { n (\%) }\end{array}$ & \\
\hline All participants & & $244(63.4)$ & $141(36.6)$ & \\
\hline \multicolumn{5}{|c|}{ Maternal age (years) } \\
\hline$\leq 24$ & $210(54.5)$ & $100(47.6)$ & $110(52.4)$ & 0.027 \\
\hline$>24$ & $175(45.5)$ & $144(82.3)$ & $31(17.7)$ & \\
\hline \multicolumn{5}{|c|}{ Maternal education level } \\
\hline Illiterate & $66(17.2)$ & $5(7.6)$ & $61(92.4)$ & $<0.001$ \\
\hline Primary & $72(18.7)$ & $61(84.7)$ & $11(18.3)$ & \\
\hline SSC or above & $247(64.2)$ & $178(72.1)$ & $69(38.9)$ & \\
\hline \multicolumn{5}{|c|}{ Maternal occupation } \\
\hline Housewife & $273(70.9)$ & 209 (76.6) & $64(23.4)$ & 0.002 \\
\hline Service holder & $112(29.1)$ & 35 (31.3) & 77 (68.8) & \\
\hline \multicolumn{5}{|c|}{ Family monthly income (BDT) } \\
\hline$<15000$ & $276(71.7)$ & $160(57.9)$ & $116(42.1)$ & $<0.001$ \\
\hline$\geq 15000$ & $109(28.3)$ & $84(77.1)$ & $25(22.9)$ & \\
\hline \multicolumn{5}{|l|}{ Family type } \\
\hline Single & $233(60.5)$ & $183(78.5)$ & $50(21.5)$ & $<0.001$ \\
\hline Joint & $152(39.5)$ & $61(40.1)$ & $91(59.9)$ & \\
\hline \multicolumn{5}{|l|}{ Religion } \\
\hline Muslim & $335(87.0)$ & $238(61.8)$ & $97(41.6)$ & \\
\hline Non-muslim & $50(12.9)$ & $06(12)$ & $44(88.0)$ & 0.268 \\
\hline \multicolumn{5}{|c|}{ Marital status of mother } \\
\hline Married & $297(77.1)$ & $235(79.1)$ & $62(20.9)$ & $<0.001$ \\
\hline Divorced & $88(22.9)$ & $9(10.2)$ & $79(89.8)$ & \\
\hline \multicolumn{5}{|l|}{ Live birth } \\
\hline 1 child & $204(52.9)$ & $161(78.9)$ & $43(21.1)$ & $<0.001$ \\
\hline 2 or more children & $181(47.01)$ & $83(45.9)$ & $98(54.1)$ & \\
\hline \multicolumn{5}{|l|}{ Place of delivery } \\
\hline Home & $122(31.7)$ & $47(38.5)$ & $75(61.5)$ & 0.008 \\
\hline Hospital & $263(68.3)$ & $197(74.9)$ & $66(25.1)$ & \\
\hline \multicolumn{5}{|l|}{ Mode of delivery } \\
\hline Normal/vaginal & $213(55.3)$ & $181(84.9)$ & $32(15.0)$ & $<0.001$ \\
\hline Caesarian & $172(44.7)$ & 63 (36.7) & $109(63.4)$ & \\
\hline
\end{tabular}

BDT: 1000 Bangladeshi Taka about US $\$ 12 .{ }^{*}$ Statistically significant: $\mathrm{p}<0.05$ (chi-squared). 
Table 2. The association of maternal knowledge and attitude scores on exclusive breastfeeding

\begin{tabular}{|c|c|c|c|c|c|}
\hline & \multirow{2}{*}{$\begin{array}{c}\text { Total } \\
\text { Yes } \\
\text { n (\%) }\end{array}$} & \multicolumn{2}{|c|}{ Exclusive breastfeeding } & \multicolumn{2}{|c|}{ Chi-squared statistics } \\
\hline & & $\begin{array}{c}\text { Yes } \\
(n=189) \\
n(\%)\end{array}$ & $\begin{array}{c}\text { No } \\
(n=111) \\
n(\%)\end{array}$ & $\chi^{2}(\mathrm{df})$ & $\mathbf{p}^{*}$ \\
\hline \multicolumn{6}{|c|}{ Knowledge score (out of 10) } \\
\hline Good & $225(58.6)$ & $171(76)$ & $54(24)$ & & \\
\hline Medium & $122(31.8)$ & $56(49.9)$ & $66(54.1)$ & $37.186(2)$ & $<0.001$ \\
\hline Poor & $38(9.1)$ & $17(44.7)$ & $21(55.3)$ & & \\
\hline \multicolumn{6}{|c|}{ Attitude score (out of 10 ) } \\
\hline Positive & $212(55.2)$ & $140(66.1)$ & $72(33.9)$ & & \\
\hline Moderate & $139(36.2)$ & $85(61.2)$ & $54(38.9)$ & $12.672(1)$ & 0.002 \\
\hline Poor & $34(8.9)$ & $12(35.3)$ & $22(64.7)$ & & \\
\hline
\end{tabular}

Knowledge score: good=7-10, medium $=4-6$, and poor $=0-3$. Attitude score: positive $=7-10$, moderate $=4-6$, and poor $=0-3$. $*$ Statistically significant: $<<0.001$ (chi-squared).

educated and the remaining $64.2 \%$ had secondary or higher level of education and most $(87.1 \%)$ of the mothers were Muslims. Most of the respondents (70.9\%) had a monthly family income <15000 BDT (1000 Bangladeshi Taka about US\$12). About half (52.9\%) of the mothers had one child and most $(77.1 \%)$ of the mothers were married. Most mothers $(70.9 \%)$ were housewives and more than half $(60.5 \%)$ of the mothers came from a single family. About half of the mothers (55.4\%) delivered their child by vaginal/normal delivery and two-thirds (68.3\%) delivered their child in hospital.

The association of maternal knowledge and attitude score on exclusive breastfeeding is shown in Table 2 . Out of 384 mothers, more than half $(63.4 \%)$ practiced exclusive breastfeeding. The mean $( \pm S D)$ knowledge and attitude scores, out of a maximum of 10 , were $7.46 \pm 2.56$ and 7.57 \pm 2.43 , respectively. More than half $(58.6 \%)$ of the mothers had a high knowledge score and about $55.2 \%$ had a positive attitude. Chi-squared test found that maternal knowledge score $\left(\chi^{2}=37.186, \mathrm{p}<0.001\right)$ and attitude score $\left(\chi^{2}=12.672\right.$, $\mathrm{p}<0.001$ ) were statistically associated with exclusive breastfeeding practices among mothers.

Table 3 shows the multiple logistic regression analysis used to identify some sociodemographic and other factors associated with exclusive breastfeeding practices among mothers. The adjusted regression model found the following sociodemographic factors were statistically significant with exclusive breastfeeding practice among mothers: housewife (AOR=5.84; 95\% CI: 2.42-14.13, p<0.001), maternal education level of primary $(\mathrm{AOR}=8.84 ; 95 \% \mathrm{CI}$ : 1.25-62.66, $\mathrm{p}<0.05$ ) and secondary or above (AOR=7.16; 95\% CI: $1.3-39.36, \mathrm{p}<0.05)$, having family monthly income $\geq 15000$ BDT $(A O R=5.41 ; 95 \%$ CI: 1.78-16.47, p<0.001), having one child (AOR=3.96; 95\% CI: 1.52-10.35, p<0.001), normal delivery (AOR=13.58; 95\% CI: 5.29-35.48, $\mathrm{p}<0.001$ ) and delivery at hospital $(\mathrm{AOR}=3.38 ; 95 \% \mathrm{CI}: 1.24-9.23$,
Table 3. Effect of sociodemographic and other characteristic on practices of exclusive breastfeeding

\begin{tabular}{l|c|c|c|}
\hline Factors & $\begin{array}{c}\text { EBF } \\
\text { Yes } \\
\text { n (\%) }\end{array}$ & AOR (95\% CI) & $\mathbf{p}^{*}$ \\
$\begin{array}{l}\text { Maternal age } \\
\text { (years) }\end{array}$ & & & \\
$\leq 24$ & $210(54.5)$ & $0.61(0.23-1.61)$ & 0.318 \\
$>24$ (Ref.) & $175(45.5)$ & - &
\end{tabular}

Maternal
education level

$\begin{array}{llll}\text { Primary } & 72(18.7) & 8.84(1.25-62.66) & \mathbf{0 . 0 2 9}\end{array}$

SSC or above $\quad 247(64.2) \quad 7.16(1.3-39.36) \quad \mathbf{0 . 0 2 4}$

\section{Maternal occupation}

Housewife $273(70.9) \quad 5.84(2.42-14.13) \quad<\mathbf{0 . 0 0 1}$

Service holder 112 (29.1)

(Ref.)

\section{Family monthly}

income (BDT)

$\begin{array}{lccc}<15000 \text { (Ref.) } & 276(71.7) & - & \\ \geq 15000 & 109(28.3) & 5.41(1.78-16.47) & \mathbf{0 . 0 0 3}\end{array}$

\section{Family type}

Single (Ref.)

Joint

233 (60.5)

\section{Marital status}

of mother

$\begin{array}{lrcc}\text { Married (Ref.) } & 297(77.1) & - & \\ \text { Divorced } & 88(22.9) & 0.12(0.03-0.58) & \begin{array}{c}\mathbf{0 . 0 0 8} \\ \text { Continued }\end{array}\end{array}$


Table 3. Continued

\begin{tabular}{|c|c|c|c|}
\hline Factors & $\begin{array}{c}\text { EBF } \\
\text { Yes } \\
\mathbf{n}(\%)\end{array}$ & AOR $(95 \% \mathrm{CI})$ & $\mathbf{p}^{*}$ \\
\hline \multicolumn{4}{|l|}{ Live birth } \\
\hline 1 Child & 204 (52.9) & $3.96(1.52-10.35)$ & 0.005 \\
\hline $\begin{array}{l}2 \text { or more } \\
\text { children (Ref.) }\end{array}$ & $181(47.01)$ & - & \\
\hline \multicolumn{4}{|l|}{$\begin{array}{l}\text { Place of } \\
\text { delivery }\end{array}$} \\
\hline Home (Ref.) & $122(31.7)$ & - & \\
\hline Hospital & $263(68.3)$ & 3.38 (1.24-9.23) & 0.017 \\
\hline \multicolumn{4}{|l|}{ Mode of delivery } \\
\hline Normal delivery & 213 (55.3) & $13.58(5.29-35.48)$ & $<0.001$ \\
\hline Caesarian (Ref.) & 172 (44.7) & - & \\
\hline
\end{tabular}

BDT: 1000 Bangladeshi Taka about US\$12. AOR: adjusted odds ratio. EBF: exclusive breastfeeding. Multiple logistic model fitted by Hosmer and Lemeshow test $\left(\chi^{2}=9\right.$, $\mathrm{df}=8, \mathrm{p}=0.67$ ). ${ }^{*}$ Statistically significant: $\mathrm{p}<0.05$ (chi-squared).

$\mathrm{p}<0.001$ ); these mothers were more likely to have good exclusive breastfeeding practice than their counterparts and it was statistically significant $(\mathrm{p}<0.05)$. Moreover, mothers from joint family (AOR=0.27; 95\% CI: 0.12-0.62, p<0.05) or divorced (AOR=0.12; 95\% CI: 0.03-0.58, $\mathrm{p}<0.005)$ were less likely to have exclusive breastfeeding practice compared to their counterparts and these factors also were statistically significant $(\mathrm{p}<0.05)$.

\section{DISCUSSION}

This study demonstrated that about two-thirds of mothers $(63.4 \%)$ exclusive breastfed their child up to 6 months of age, which is close to the national level (65\%) in Bangladesh ${ }^{13}$. The prevalence of EBF in this study was higher than other studies conducted in Bangladesh such as a study conducted among Bangladeshi mothers in a rural area which reported a prevalence of EBF of $35.9 \%{ }^{15}$ and another study conducted in the rural area of Rajshahi district which reported an EBF practice rate of $34.5 \%{ }^{16}$. In both studies, the rural areas lacked health facilities and a large proportion of participants was illiterate. EBF practice rate was relatively lower among illiterate mothers compared with literate mothers as found in another study ${ }^{17}$. So, health facilities and educational level were associated with lower EBF practice in that study area. In this study, the prevalence of EBF reported could be attributed to the large number of educated mothers, availability of health facilities, and presence of NGOs implementing programs aimed at improving breastfeeding and reducing child malnutrition.

This study found that a good knowledge score on breastfeeding was statistically significant with EBF practice among mothers, in accordance with previous studies ${ }^{3,18}$. A recent study conducted in Wajir, Kenya, reported that knowledge score was not associated with EBF practice, in contrast with our findings ${ }^{14}$. In that study, high knowledge was attributed to the presence of government and NGO intervention programs aimed at improving infant and young child feeding (IYCF) practices to achieve the aims of the National Nutrition Action Plan of Kenya. WHO model of IYCF behavior reports that cultural attitudes, beliefs and norms are important factors associated with infant formula feeding ${ }^{19}$. Cultural beliefs on infant feeding may result in more infant formula feeding than EBF practice. So, cultural beliefs were identified that may lead to failed EBF practices. However, a study conducted among Nigerian ${ }^{3}$ mothers found that a good knowledge score indicates better EBF practice, which was similar to our study. Furthermore, a study conducted among Jamaican mothers ${ }^{18}$ reported that the EBF practices were significantly considered by those who had adequate knowledge on breastfeeding. Good knowledge score indicates awareness of the advantages of exclusive breastfeeding and the disadvantages of formula milk/bottle feeds.

The present study revealed that a positive attitude towards breastfeeding significantly improved EBF practice among mothers, consistent with previous studies ${ }^{14,18}$. However, previous studies conducted among Jamaican mothers ${ }^{18}$ and among Kenyan mothers ${ }^{14}$ found a similarly significant relationship between EBF practices and a positive attitude of the mother on breastfeeding. Mothers who were positive toward exclusive breastfeeding agreed that breastfeeding is beneficial both for infant and mother, that the baby can survive without water up to 6 months of age, formula milk is unsuitable for baby and not as easily digested as breast milk.

In contrast, this study found that some sociodemographic factors such as being a housewife, literate, having a high income, and being a single family, were more likely to practice EBF. Previous studies conducted among Malaysian ${ }^{20}$ and Indian mothers ${ }^{21}$ reported that non-working/housewife mothers were more likely to have good EBF practices than employed mothers who had poor knowledge and attitude toward EBF practice, leading to less exclusive breastfeeding practices, as found among Nepali ${ }^{22}$ and Bangladeshi ${ }^{16}$ mothers. Housewives can easily participate in Government and NGO implemented programs on Infant and Young Feeding (IYCF) practices available in the community, which makes them more positive toward EBF practices. Moreover, a study conducted in Indian mothers ${ }^{17}$ demonstrated that EBF practice rate was high in educated mothers, which is similar to our study findings. Educated mothers know about the advantages of exclusive breastfeeding and disadvantages of formula milk/bottle feeds, from school-based interventions promoting exclusive breastfeeding. Furthermore, mothers with family monthly income $\geq 15000$ BDT were more likely to have good practices on exclusive breastfeeding than those with $<15000$ BDT, inconsistent with the results found in another study ${ }^{16}$. Previous study assumptions were that most 
of the low monthly income mothers watched BTV. Many Governments and NGOs choose BTV as a good medium for broadcasting different events to promote breastfeeding practices. At present, different social media such as Facebook, Twitter etc., have comparatively more users among rich families and Government and NGOs implement their interventions to promote exclusive breastfeeding through these media and also provide videos about the advantages of breastfeeding and disadvantages of breast milk substitutes. Moreover, mothers from joint families, compared to mothers from single families, are less likely to exclusive breastfeeding practices, consistent with results not only found in $\mathrm{Nepal}^{22}$ but also in India ${ }^{17}$. So, the early cessation of EBF among mothers in a joint family compared to those living in single family needs further investigation to understand the sociodemographic factors underlying the family structures and EBF practices.

This study found few health service-related factors such as types and mode of delivery associated with exclusive breastfeeding. However, women with caesarian delivery were less likely to practice EBF compared to those with vaginal delivery; similar results were found in previous studies ${ }^{21,22}$. Caesarian mothers required more time to recovery from cesarean illness (fatigue, low blood pressure, unable to practice breastfeeding) and difficulty in moving; reasons why families were likely to introduce formula milk and not exclusive breastfeeding ${ }^{12}$. Furthermore, it was found that hospital delivery mothers had good EBF practices compared with home delivery mothers. Study results were consistent with a study in Northwest Ethiopia ${ }^{23}$. Delivery in hospital made counseling easier to prepare mother to be more positive toward EBF practices by duty doctor/nurse. In this study area, this program implements the Bangladesh Breastfeeding Foundation, part of the Ministry of Health and Institute of Public Health and Nutrition services, which might affect the study findings.

There are two main findings in this study. First, higher knowledge and better attitude were associated with more likelihood to follow EBF practices among study participants. Second, literate, housewife mothers, and mothers who delivered their child through normal/vaginal birth and delivery at hospital were more likely to practice EBF.

\section{Strengths and limitations}

The strength of this study is that it was the first study in Dhaka, Bangladesh, to report that knowledge, attitude and other factors were associated with exclusive breastfeeding and explored current exclusive breastfeeding status. This study had also some limitations such as it was a crosssectional, hospital outdoor-based study that does not allow any complete progressive association identifying knowledge, attitude and sociodemographic parameters on exclusive breastfeeding. Additionally, information on the reasons for discontinuing exclusive breastfeeding was not collected. Another limitation of this study is that it did not measure perceived insufficient milk, the major risk factor for early breastfeeding discontinuation globally. Further longitudinal research is needed to explore the effects of different sociodemographic factors on exclusive breastfeeding.

\section{CONCLUSIONS}

This study found several factors, such as good knowledge and positive attitude, hospital and vaginal delivery, education level, and housewife mothers, were significantly associated with good exclusive breastfeeding practices. These findings help to design interventions that need to improve exclusive breastfeeding. Counselling programs both at the community and individual level to promote exclusive breastfeeding practices are needed. Government and NGOs should take steps designing an intervention on discouraging home delivery in absence of healthcare providers or skilled birth attendant, and encouraging vaginal delivery to increase exclusive breastfeeding practices among Bangladeshi mothers. Initiatives should be taken for the proper execution of the recommended interventions, which should significantly increase EBF practice, reduce infant morbidity and mortality rates, and help to achieve SDG-3 in Bangladesh.

\section{REFERENCES}

1. World Health Organization. Global strategy for infant and young child feeding: The optimal duration of exclusive breastfeeding. Fifthy-fourth World Health Assembly. Document number A54/INF.DOC./4. May 1, 2001. Accessed July 10, 2021. https://apps.who.int/gb/archive/pdf_files/ WHA54/ea54id4.pdf

2. Amele EA, Demissie BW, Desta KW, Woldemariam EB. Prelacteal feeding practice and its associated factors among mothers of children age less than 24 months old in Southern Ethiopia. Ital J Pediatr. 2019;45(1):15. doi:10.1186/s13052-019-0604-3

3. Akinyinka M. Breastfeeding Knowledge and Practices among Mothers of Children under 2 Years of Age Living in a Military Barrack in Southwest Nigeria. Int J MCH AIDS. 2016;5(1):113. Accessed July 10, 2021. https://www.ncbi.nlm.nih.gov/ pmc/articles/PMC5005982/pdf/IJMA-5-1.pdf

4. World Health Organization, UNICEF. Global Breastfeeding Scorecard, 2018: Enabling Women To Breastfeed Through Better Policies And Programmes. World Health Organization, Unicef; 2018. Accessed July 10, 2021. https://www.who. int/nutrition/publications/infantfeeding/global-bfscorecard-2018.pdf?ua $=1$

5. Global breastfeeding scorecard. Global Breastfeeding Collective. Accessed July 10, 2021. https://www. globalbreastfeedingcollective.org/about-collective

6. UNICEF, World Health Organization. Capture the Moment - Early initiation of breastfeeding: The best start for every newborn. UNICEF, World Health Organization; 2018. Accessed July 10, 2021. https://www.unicef.org/eca/ media/4256/file/Capture-the-moment-EIBF-report.pdf

7. National Institute of Population Research and Training, Mitra 
and Associates, ICF International. Bangladesh Demographic and Health Survey 2014. March 2016. Accessed July 10, 2021. https://dhsprogram.com/pubs/pdf/FR311/FR311.pdf

8. Karim F, Billah SM, Chowdhury MAK, et al. Initiation of breastfeeding within one hour of birth and its determinants among normal vaginal deliveries at primary and secondary health facilities in Bangladesh: A case-observation study. PLoS One. 2018;13(8):e0202508. doi:10.1371/journal.pone.0202508

9. Sen KK, Mallick TS, Bari W. Gender inequality in early initiation of breastfeeding in Bangladesh: A trend analysis. Int Breastfeed J. 2020;15:18. doi:10.1186/s13006-020-00259-y

10. Goga AE, Doherty T, Jackson DJ, et al. Infant feeding practices at routine PMTCT sites, South Africa: results of a prospective observational study amongst HIV exposed and unexposed infants - birth to 9 months. Int Breastfeed J. 2012;7:4. doi:10.1186/1746-4358-7-4

11. Siri BAA, Bengaly M, Garanet F, et al. Knowledge, Opinions and Attitudes of Mothers About Breastfeeding and Child Feeding in Rural Areas of Burkina Faso: A Study in Ouargaye's District Health Facilities. Journal of Family Medicine and Health Care. 2018;4(3):13-19. doi:10.11648/j. jfmhc.20180403.11

12. Joshi PC, Angdembe MR, Das SK, Ahmed S, Faruque ASG, Ahmed T. Prevalence of exclusive breastfeeding and associated factors among mothers in rural Bangladesh: a cross-sectional study. Int Breastfeed J. 2014;9:7. doi:10.1186/1746-4358-9-7

13. National Institute of Population Research and Training, ICF. Bangladesh Demographic and Health Survey 2017-18: Key Indicators. National Institute of Population Research and Training, ICF; 2019. Accessed July 10, 2021. https:// dhsprogram.com/pubs/pdf/PR104/PR104.pdf

14. Mohamed MJ, Ochola S, Owino VO. Comparison of knowledge, attitudes and practices on exclusive breastfeeding between primiparous and multiparous mothers attending Wajir District hospital, Wajir County, Kenya: a cross-sectional analytical study. Int Breastfeed J.

\section{8;13:11. doi:10.1186/s13006-018-0151-3}

15. Hossain M, Islam A, Kamarul T, Hossain G. Exclusive breastfeeding practice during first six months of an infant's life in Bangladesh: a country based cross-sectional study. BMC Pediatr. 2018;18(1):93. doi:10.1186/s12887-018-1076-0

16. Rana MM, Islam MR, Karim MR, et al. Knowledge and practices of exclusive breastfeeding among mothers in rural areas of Rajshahi district in Bangladesh: A community clinic based study. PLoS One. 2020;15(5):e0232027. doi:10.1371/journal.pone.0232027

17. Velusamy V, Premkumar PS, Kang G. Exclusive breastfeeding practices among mothers in urban slum settlements: pooled analysis from three prospective birth cohort studies in South India. Int Breastfeed J. 2017;12:35. doi:10.1186/s13006-017-0127-8

18. Chatman LM, Salihu HM, Roofe ME, Wheatle P, Henry D, Jolly PE. Influence of Knowledge and Attitudes on Exclusive Breastfeeding Practice Among Rural Jamaican Mothers. Birth. 2004;31(4):265-271. doi:10.1111/j.0730-7659.2004.00318.x

19. Wanjohi M, Griffiths P, Wekesah F, et al. Sociocultural factors influencing breastfeeding practices in two slums in Nairobi, Kenya. Int Breastfeed J. 2017;12:5. doi:10.1186/s13006-016-0092-7

20. Tan KL. Factors associated with exclusive breastfeeding among infants under six months of age in peninsular malaysia. Int Breastfeed J. 2011;6:2. doi:10.1186/1746-4358-6-2

21. Pal AC, Mukhopadhyay DK. Knowledge, Attitude and Practice of Breastfeeding in a Rural Community of Bankura District, West Bengal, India. IOSR journal of dental and medical sciences. 2014;13(11):24-28. doi:10.9790/0853-131172428

22. Ulak M, Chandyo RK, Mellander L, Shrestha PS, Strand TA. Infant feeding practices in Bhaktapur, Nepal: a crosssectional, health facility based survey. Int Breastfeed J. 2012;7:1. doi:10.1186/1746-4358-7-1

23. Arage G, Gedamu H. Exclusive Breastfeeding Practice and Its Associated Factors among Mothers of Infants Less Than Six Months of Age in Debre Tabor Town, Northwest Ethiopia: A Cross-Sectional Study. Adv Public Health. 2016;2016:3426249. doi:10.1155/2016/3426249

\section{ACKNOWLEDGEMENTS}

We thank our study participants who answered all our research questions and gave their valuable time. The authors thank the executive director of the hospital and are grateful to Mohsina Hossain Mita (students of Faculty of Nutrition and Food Science, Patuakhali Science and Technology University) that helped during data collection process.

\section{CONFLICTS OF INTEREST}

The authors have completed and submitted the ICMJE Form for Disclosure of Potential Conflicts of Interest and none was reported.

FUNDING

There was no source of funding for this research.

\section{ETHICAL APPROVAL AND INFORMED CONSENT}

This study was conducted in accordance with Bangladesh Medical Association Act (BMA) and following the guidelines of the Helsinki Declaration 1975. Moreover, ethical approval was obtained from the Research Ethical Committee (REC) of the Department of Environmental Sanitation, Patuakhali Science and Technology University, Bangladesh
(Approval No. ENS: 26/05/2019:09). The purpose of the study was explained in detail to the participants and written informed consent was obtained from the subjects before participation in the study. Moreover, permission was obtained from the Executive Director of the Mother and Child Hospital, Dhaka, Bangladesh.

\section{DATA AVAILABILITY}

The data supporting this research are available from the corresponding author on reasonable request.

\section{AUTHORS' CONTRIBUTIONS}

MH: study concept and design, data analysis, methodology and writing original draft. NH: reviewing, editing and supervising the study. SIK: revision of the draft manuscript for important intellectual content. MAT: Critical revision of the final manuscript. MSA: data collection and analysis. All authors approved the final manuscript.

\section{PROVENANCE AND PEER REVIEW}

Not commissioned; externally peer reviewed. 